\title{
Efficacy and safety of Xa inhibitors in patients with heart failure and coronary or peripheral artery disease: a systematic review and meta-analysis of randomized controlled trials
}

\author{
Yuqiao Chen ${ }^{1}$, Xiu Feng ${ }^{2}$, Chenliang Qi $^{1}$, Lingfeng Zhou ${ }^{1}$, Yi Sun ${ }^{1}$, Zhenhua Gu ${ }^{1}$, Xiaolong Li $^{1}$ \\ ${ }^{1}$ Department of Cardiology, Changzhou Hospital of Traditional Chinese Medicine, Changzhou, China; ${ }^{2}$ Department of Echocardiography, The First \\ People's Hospital of Changzhou, Changzhou, China \\ Contributions: (I) Conception and design: Y Chen, X Feng, X Li; (II) Administrative support: X Li; (III) Provision of study materials or patients: \\ X Feng, C Qi, L Zhou; (IV) Collection and assembly of data: Y Chen, X Feng, C Qi, Y Sun; (V) Data analysis and interpretation: Y Chen, X Feng, \\ Z Gu, X Li; (VI) Manuscript writing: All authors; (VII) Final approval of manuscript: All authors. \\ Correspondence to: Xiaolong Li. Department of Cardiology, Changzhou Hospital of Traditional Chinese Medicine, No. 25, Heping North Road, \\ Changzhou 213000, China. Email: 1x118661138721@163.com.
}

\begin{abstract}
Background To evaluate the efficacy and safety of Xa inhibitors in patients with heart failure (HF) and coronary artery disease (CAD) or peripheral artery disease (PAD).

Methods: A systematic electronic literature search was performed using the PubMed, Web of Science, EMBASE, and Cochrane Library databases from inception to June 26, 2019. A total of four randomized controlled trials involving 14,694 patients were included in this meta-analysis.

Results: The meta-analysis showed that there was no statistical difference between the Xa inhibitor and control group regarding the primary efficacy outcome [rivaroxaban $2.5 \mathrm{mg}$ group: relative risk (RR) 0.82 , 95\% CI: 0.66-1.01, P=0.06; rivaroxaban $5 \mathrm{mg}$ group: RR 0.86, 95\% CI: 0.73-1.02, P=0.08]. The risk of the primary safety outcome was significantly increased among patients who received Xa inhibitors compared with the control group (rivaroxaban $2.5 \mathrm{mg}$ group: RR 1.55, 95\% CI: $1.21-1.98, \mathrm{P}=0.0006$; rivaroxaban $5 \mathrm{mg}$ group: RR 1.66, 95\% CI: 1.30-2.12, $\mathrm{P}<0.0001)$. There was no significant difference in the risk of cardiovascular death between the Xa inhibitor and control group (rivaroxaban $2.5 \mathrm{mg}$ group: RR 0.79, 95\% CI: 0.54-1.14, $\mathrm{P}=0.21$; rivaroxaban $5 \mathrm{mg}$ group: RR 0.89, 95\% CI: $0.73-1.08, \mathrm{P}=0.24)$. The risk of myocardial infarction (MI) in the rivaroxaban $5 \mathrm{mg}$ group was significantly lower than that of the control group (RR 0.83, 95\% CI: 0.69-0.99, P=0.04). However, the risk of $\mathrm{MI}$ in the rivaroxaban $2.5 \mathrm{mg}$ group was similar to that of the control group (RR 0.85, 95\% CI: $0.71-1.01, \mathrm{P}=0.07$ ).

Discussion: $\mathrm{Xa}$ inhibitors were associated with a higher risk of major adverse cardiovascular events and bleeding among HF and CAD or PAD patients. Therefore, Xa inhibitors should be used cautiously in patients with $\mathrm{HF}$ and $\mathrm{CAD}$ or PAD.
\end{abstract}

Keywords: Xa inhibitor; heart failure (HF); coronary artery disease (CAD); peripheral artery disease (PAD); metaanalysis

Submitted May 27, 2021. Accepted for publication Jul 14, 2021.

doi: 10.21037/apm-21-1645

View this article at: https://dx.doi.org/10.21037/apm-21-1645

\section{Introduction}

Heart failure (HF) is an important cause of human morbidity and mortality and is considered an epidemic disease worldwide, affecting approximately $1 \%$ to $3 \%$ of the adult population (1). Because of the abnormality of the clotting cascade and blood flow in patients with HF, thrombotic complications (including ischemic stroke, peripheral arterial embolism, and pulmonary embolism, 
etc.) are common among HF patients (2). HF and coronary artery disease (CAD) usually coexist in clinical practice. Coronary arteries with thrombosis are more prone to myocardial infarction (MI) or sudden death (3). Therefore, oral anticoagulants (OACs) are essential in the management of $\mathrm{HF}$ with atrial fibrillation (AF) (4).

Recently, prospective evidence from four randomized controlled trials, the WASH (5), WATCH (6), HELAS (7), and WARCEF (8) trials, for vitamin K antagonist (VKA) efficacy in patients with HF and sinus rhythm has become available. These four trials showed that there was no significant difference in benefit events other than ischemic stroke between the VKA group and the other groups. Furthermore, patients in the VKA group may have higher rates of major hemorrhages (9). Therefore, the clinical application of OACs remains controversial among patients with $\mathrm{HF}$ and $\mathrm{CAD}$ or PAD. Fortunately, Xa inhibitors are a new type of OAC that is being used widely clinically, because it significantly improves compliance and decreases bleed complications. Therefore, $\mathrm{Xa}$ inhibitors are promising OACs for the treatment of patients with $\mathrm{HF}$ and $\mathrm{CAD}$ or PAD.

Most previous studies focused on patients with HF complicated with AF. And the application value of OACs in AF was relatively clear. Patients with HF combined with CAD and PAD are high-risk groups for cardiocerebrovascular adverse events. However, there is limited information about the efficacy and safety of Xa inhibitors among patients with HF combined with CAD or PAD. Therefore, we conducted this meta-analysis to evaluate the efficacy and safety of Xa inhibitors in patients with HF combined with CAD or PAD. We present the following article in accordance with the PRISMA reporting checklist (available at https://dx.doi.org/10.21037/apm-21-1645).

\section{Methods}

\section{Search strategy and study selection}

Two independent reviewers (YQC and XF) conducted a comprehensive literature search using a search strategy combining $\mathrm{MeSH}$ terms and free word searches in the PubMed, Web of Science, EMBASE, and Cochrane Library databases from inception to June 26, 2019. We identified randomized clinical trials (RCTs) that evaluated the efficacy and safety of Xa inhibitors in patients with HF and $\mathrm{CAD}$ or PAD. The search terms used were (CAD OR PAD) AND HF AND Xa inhibitors [details summarized in the Supplementary file (Appendix 1)]. After excluding duplicated records, titles and abstracts were screened, and then entire articles were assessed based on inclusion and exclusion criteria. Data were extracted following the Preferred Reporting Items for Systematic reviews and Meta-Analyses (PRISMA) guidelines (10).

\section{Inclusion and exclusion criteria}

The inclusion criteria were as follows: (I) design: original articles of RCTs; (II) population: patients with HF and CAD or PAD; (III) interventions: oral Xa inhibitors with or without antiplatelet agents. The exclusion criteria were: (I) no data about efficacy and safety of patients, and (II) written in a language other than English.

\section{Data extraction}

Two reviewers (YQC and XF) independently extracted data from included studies. Disagreements were resolved through discussion with a third investigator (CLQ). The information extracted for each study included the lead author, publication year, participant characteristics, study design, sample size, and outcomes.

\section{Study quality assessment}

Assessment for risk of bias was independently conducted by two authors (LFZ and YS). The Cochrane Collaboration Risk of Bias Tool was used to assess the risk of bias in RCTs (11), including random sequence generation, allocation concealment, blinding, incomplete outcome data, selective reporting, and other sources of bias. Publication bias was not assessed, as the total number of included studies was less than 10 .

\section{Efficacy and safety outcomes}

The primary efficacy outcome was the composite endpoint of death from any cause, $\mathrm{MI}$ and stroke, or the composite endpoint of death from any cardiovascular (CV) causes, MI and stroke (APPRAISE-2 trial used ischemic stroke) (12). The primary safety outcome was major bleeding defined by the International Society on Thrombosis and Hemostasis (ISTH) or major bleeding according to the Thrombolysis in Myocardial Infarction (TIMI) definition. The secondary endpoints included mortality, risk of MI, risk of stroke, etc. 


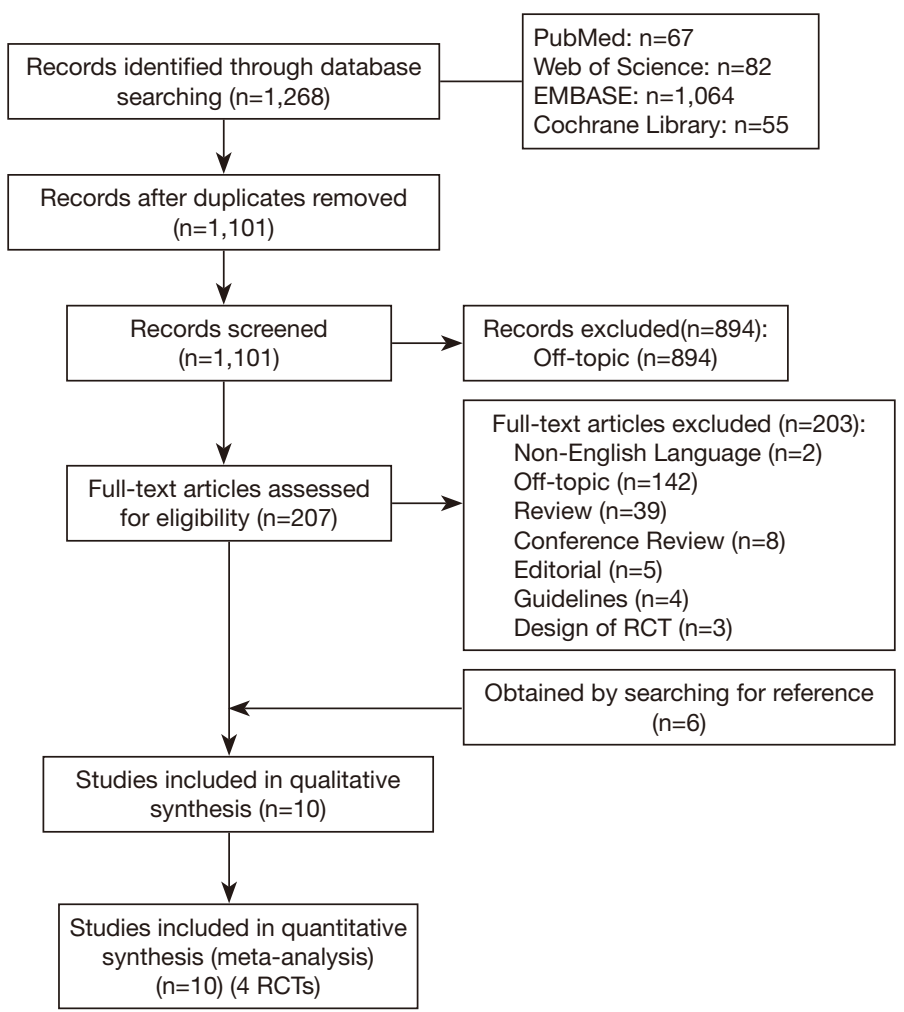

Figure 1 Flow diagram of study identification and selection process. RCT, randomized controlled trial.

\section{Statistical analysis}

The Review Manager 5.3 software (RevMan 5.3) was used to perform the analysis. Dichotomous outcomes were presented as a risk ratio (RR) and $95 \%$ confidence intervals (CIs). Studies were tested for heterogeneity using the $\mathrm{I}^{2}$ statistic, and an $\mathrm{I}^{2}$ value of greater than $50 \%$ suggested substantial heterogeneity. Fixed-effects models were used to pool data with insignificant heterogeneity and randomeffects models for data with significant heterogeneity. A sensitivity analysis was performed by excluding one trial at a time to test the influence of a single study on the overall pooled estimate. Subgroup analysis was performed for patients with and without acute coronary syndrome (ACS). The significance level was set at a $\mathrm{P}$ value of 0.05 .

\section{Results}

\section{Study identification and selection}

The electronic literature search strategy yielded 1,268 records, of which 167 were excluded as duplicates, and 894 were considered off-topic after an initial screen of title and abstract, as they did not explicitly address Xa inhibitor use in patients with $\mathrm{HF}$ and $\mathrm{CAD}$ or PAD. Another 203 articles were excluded after a full-text review. Based on the inclusion and exclusion criteria, 4 eligible articles (12-15) were included, and another 6 articles (16-21) were obtained by searching through reference lists. The final sample comprised 4 RCTs, the APPRAISE-2 (12), ATLAS ACS 2-TIMI 51 (17), COMPASS (19), and COMMANDER HF (15) trials, involving 14,694 participants for inclusion in the quantitative analysis. The details of the study identification and selection process are shown in Figure 1. Since the APPRAISE-2 trial only provided the event rate per 100 patient-years of the apixaban and placebo groups in patients with prior HF, we calculated the data for each group based on the events in the total group, assuming the same follow-up time for each group.

\section{Characterization of patients}

Of the total of 14,694 participants included in this metaanalysis, 8,589 were in the $\mathrm{Xa}$ inhibitors group (with or without antiplatelet agents) and 6,105 patients in the 
Table 1 Basic information of RCTs and baseline characteristics of patients

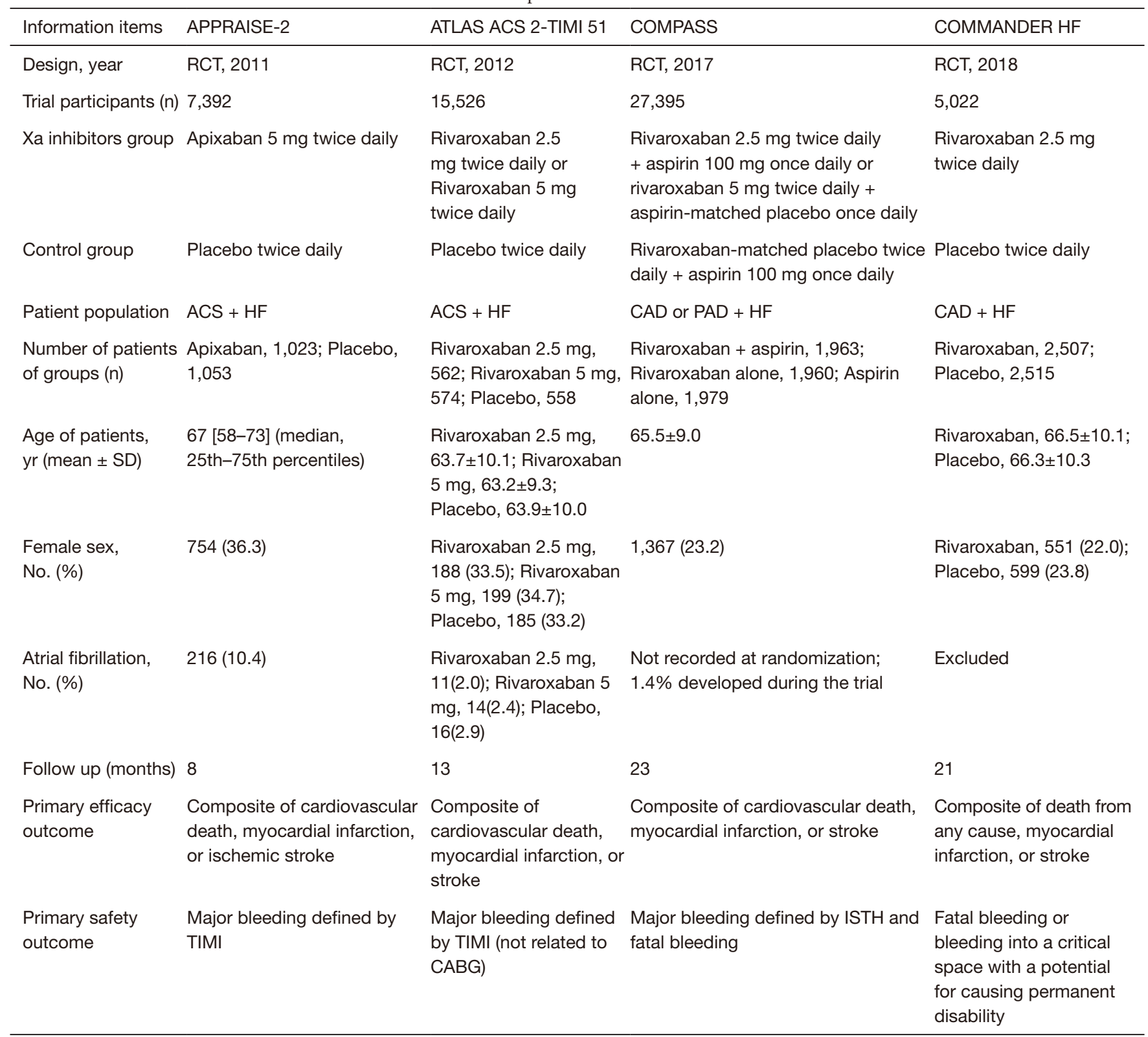

RCT, randomized controlled trial; APPRAISE-2, The Apixaban for Prevention of Acute Ischemic Events 2; ATLAS ACS 2-TIMI 51, AntiXa Therapy to Lower Cardiovascular Events in Addition to Standard Therapy in Subjects with Acute Coronary Syndrome-Thrombolysis in Myocardial Infarction 51; COMPASS, Cardiovascular Outcomes for People Using Anticoagulation Strategies; COMMANDER HF, A Study to Assess the Effectiveness and Safety of Rivaroxaban in Reducing the Risk of Death, Myocardial Infarction, or Stroke in Participants with Heart Failure and Coronary Artery Disease Following an Episode of Decompensated Heart Failure; ACS, acute coronary syndrome; $\mathrm{HF}$, heart failure; $\mathrm{CAD}$, coronary artery disease; PAD, peripheral artery disease; SD, standard deviation; TIMI, thrombolysis in myocardial infarction; CABG, coronary artery bypass graft; ISTH, International Society on Thrombosis and Hemostasis.

control group (placebo or aspirin alone). The mean followup duration ranged from 8 months to 23 months. The baseline characteristics of patients are listed in Table 1.

Participants in the APPRAISE-2 and COMMANDER
HF trials were randomly divided into the Xa inhibitor and control group, but the Xa inhibitor groups in the ATLAS ACS 2-TIMI 51 and COMPASS trials comprised two different treatment regimens. We used groups with the 


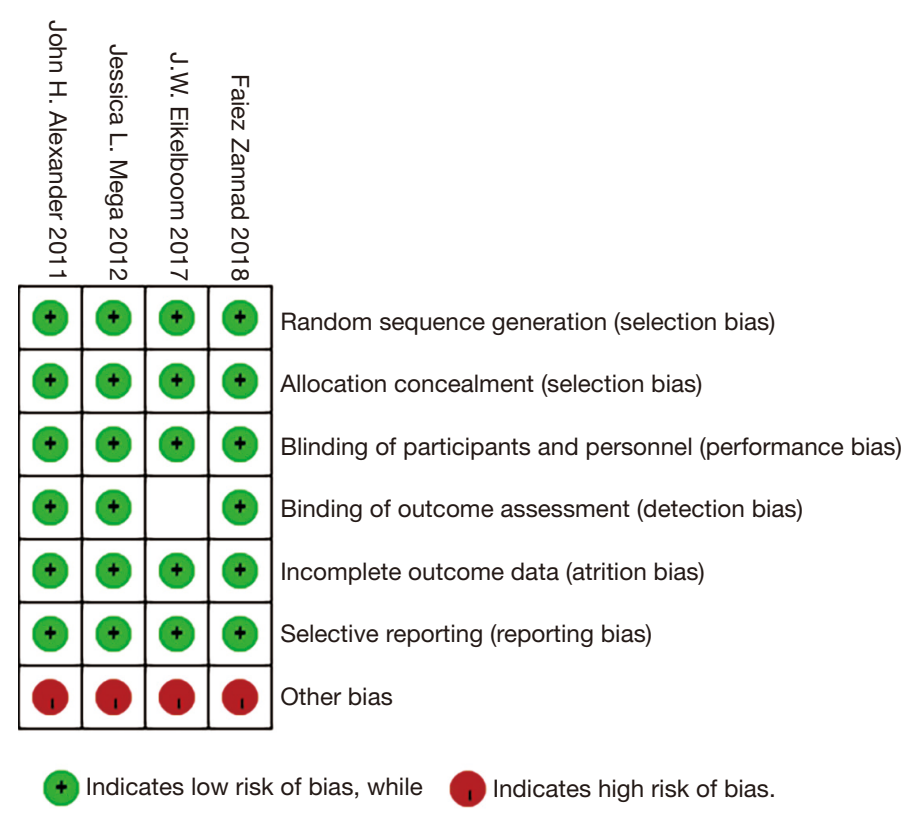

Figure 2 Risk of bias summary. Faiez Zannad 2018 corresponds to COMMANDER HF (A Study to Assess the Effectiveness and Safety of Rivaroxaban in Reducing the Risk of Death, Myocardial Infarction, or Stroke in ParticipantswithHeart Failure and Coronary Artery Disease Following an Episode of Decompensated Heart Failure) trial. J.W. Eikelboom 2017 corresponds to COMPASS (Cardiovascular Outcomes for People Using Anticoagulation Strategies) trial. Jessica L. Mega 2012 corresponds to ATLAS ACS 2-TIMI 51 (Anti-Xa Therapy to Lower Cardiovascular Events in Addition to Standard Therapy in Subjects with Acute Coronary Syndrome-Thrombolysis in Myocardial Infarction 51) trial. John H. Alexander 2011 corresponds to APPRAISE-2 (The Apixaban for Prevention of Acute Ischemic Events 2) trial. The correspondence relations are same to following figures.

same dose of $\mathrm{Xa}$ inhibitor as a parallel comparison and compared them separately with the control group. The APPRAISE-2 and ATLAS ACS 2-TIMI 51 trials enrolled patients with ACS, but no such patients were included for the COMPASS and COMMANDER HF trials. Therefore, we performed a subgroup analysis for patients with ACS.

\section{Risks of bias summary}

In this study, the recommendations of the Cochrane Handbook for Systematic Reviews of Interventions were implemented (22). The results of the assessment showed that all of the four trials had a low risk of bias. The risk of bias assessment details is shown in Figure 2.

\section{Primary efficacy outcome}

Except for the COMMANDER HF trial, which used the composite endpoint of death from any cause, MI, or stroke as the primary efficacy outcome, the other three trials defined the composite endpoint as death from $\mathrm{CV}$ events,
MI, or stroke. (In the APPRAISE-2 trial, it was an ischemic stroke, and intracranial bleeding accounted for $0.3 \%$ and $0.1 \%$ in the apixaban and placebo group, respectively). CV death accounted for about $84.3 \%(929 / 1,102)$ of deaths from any cause in the COMMANDER HF trial. There was no significant difference between the $\mathrm{Xa}$ inhibitor and control group regarding the primary efficacy outcome (rivaroxaban $2.5 \mathrm{mg}$ group: RR 0.82, 95\% CI: 0.66-1.01, $\mathrm{P}=0.06, \mathrm{I}^{2}=77 \%, \mathrm{P}$ for heterogeneity 0.004 , Figure 3 A; rivaroxaban $5 \mathrm{mg}$ group: RR 0.86, 95\% CI: 0.73-1.02, $\mathrm{P}=0.08, \mathrm{I}^{2}=62 \%, \mathrm{P}$ for heterogeneity 0.05 , Figure $\left.3 B\right)$.

\section{Primary safety outcome}

The primary safety outcome was defined as major bleeding according to the TIMI criteria in the APPRAISE-2 and ATLAS ACS 2-TIMI 51 trials. Data of major bleeding according to the ISTH criteria was reported in the COMPASS and COMMANDER HF trials. The risk of the primary safety outcome was significantly increased among patients receiving $\mathrm{Xa}$ inhibitors when compared with the 


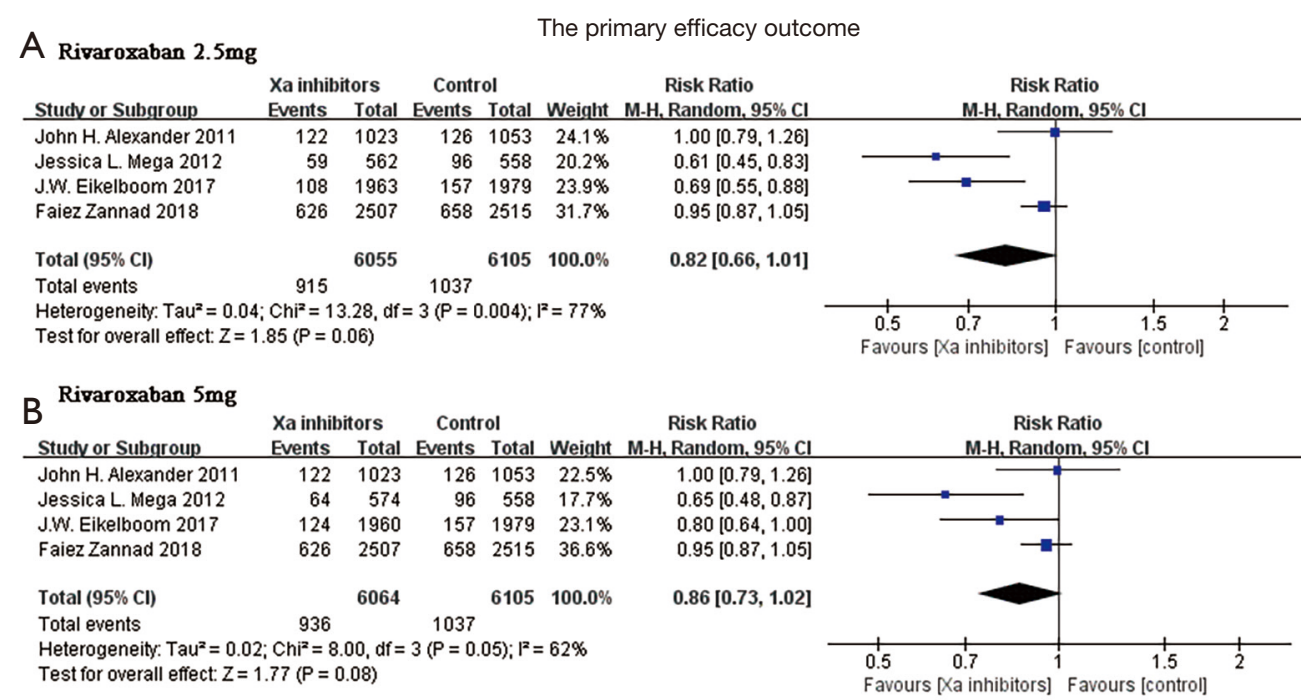

Figure 3 Meta-analysis for the primary efficacy outcome. (A) Includes the rivaroxaban $2.5 \mathrm{mg}$ group in the ATLAS ACS 2-TIMI 51 trial and rivaroxaban $2.5 \mathrm{mg}$ plus aspirin $100 \mathrm{mg}$ group in the COMPASS trial; (B) includes the rivaroxaban $5 \mathrm{mg}$ group in the ATLAS ACS 2-TIMI 51 trial and the rivaroxaban $5 \mathrm{mg}$ group in the COMPASS trial. ATLAS ACS 2-TIMI 51, Anti-Xa Therapy to Lower Cardiovascular Events in Addition to Standard Therapy in Subjects with Acute Coronary Syndrome-Thrombolysis in Myocardial Infarction 51. COMPASS, Cardiovascular Outcomes for People Using Anticoagulation Strategies.

control group (rivaroxaban $2.5 \mathrm{mg}$ group: RR 1.55, 95\% CI: $1.21-1.98, \mathrm{P}=0.0006, \mathrm{I}^{2}=0 \%, \mathrm{P}$ for heterogeneity 0.66 , Figure $4 A$; rivaroxaban $5 \mathrm{mg}$ group: RR 1.66, 95\% CI: 1.30 $2.12, \mathrm{P}<0.0001, \mathrm{I}^{2}=0 \%, \mathrm{P}$ for heterogeneity 0.97 , Figure $\left.4 B\right)$.

\section{Cardiovascular deatb}

Only the APPRAISE-2, ATLAS ACS 2-TIMI 51 and COMMANDER HF trials provided data about $\mathrm{CV}$ death. There was no statistically significant difference in the $\mathrm{CV}$ death rate between patients in the $\mathrm{Xa}$ inhibitor and control groups (rivaroxaban $2.5 \mathrm{mg}$ group: RR 0.79, 95\% CI: 0.54 $1.14, \mathrm{P}=0.21, \mathrm{I}^{2}=77 \%, \mathrm{P}$ for heterogeneity 0.01 , Figure $5 \mathrm{~A}$; rivaroxaban $5 \mathrm{mg}$ group: RR 0.89, 95\% CI: 0.73-1.08, $\mathrm{P}=0.24, \mathrm{I}^{2}=37 \%$, P for heterogeneity 0.21 , Figure $5 B$ ).

\section{Myocardial infarction}

Data on the incidence of MI among patients with $\mathrm{HF}$ and CAD or PAD was not reported in the COMPASS trial. The risk of $\mathrm{MI}$ in patients who received $2.5 \mathrm{mg}$ rivaroxaban was not significantly different from that of the control group (RR 0.85, 95\% CI: 0.71-1.01, P=0.07, $\mathrm{I}^{2}=0 \%, \mathrm{P}$ for heterogeneity 0.53 , Figure $6 A$ ). While patients who received $5 \mathrm{mg}$ rivaroxaban had a lower risk of $\mathrm{MI}(\mathrm{RR} 0.83,95 \%$
CI: $0.69-0.99, \mathrm{P}=0.04, \mathrm{I}^{2}=22 \%, \mathrm{P}$ for heterogeneity 0.28 , Figure $6 B$ ) when compared with the control group.

\section{Stroke}

There was a similar risk of stroke in the $\mathrm{Xa}$ inhibitor and the control group for both the $2.5 \mathrm{mg}$ rivaroxaban dosage (RR 0.68, 95\% CI: 0.26-1.80, $\mathrm{P}=0.44$ ) and the $5 \mathrm{mg}$ rivaroxaban dosage (RR 1.13, 95\% CI: $0.49-2.62, \mathrm{P}=0.78$ ) in the ATLAS ACS 2-TIMI 51 trial. Rivaroxaban with aspirin reduced the RR of stroke by $52 \%$ (RR $0.48,95 \%$ CI: 0.28-0.83) in patients with HF in the COMPASS trial. In the COMMANDER HF trial, a significant reduction in the risk of stroke was also found in the rivaroxaban group (RR 0.66, 95\% CI: 0.47-0.95). The risk of stroke was not significantly different in the rivaroxaban group compared to the control group in the APPRAISE-2 trial.

\section{Sensitivity analysis}

There was significant heterogeneity concerning comparisons with the primary efficacy outcome. Regarding the comparisons with the rivaroxaban $2.5 \mathrm{mg}$ groups, although heterogeneity decreased significantly $\left(I^{2}=15 \%, P\right.$ for heterogeneity 0.31) after excluding the ATLAS ACS 


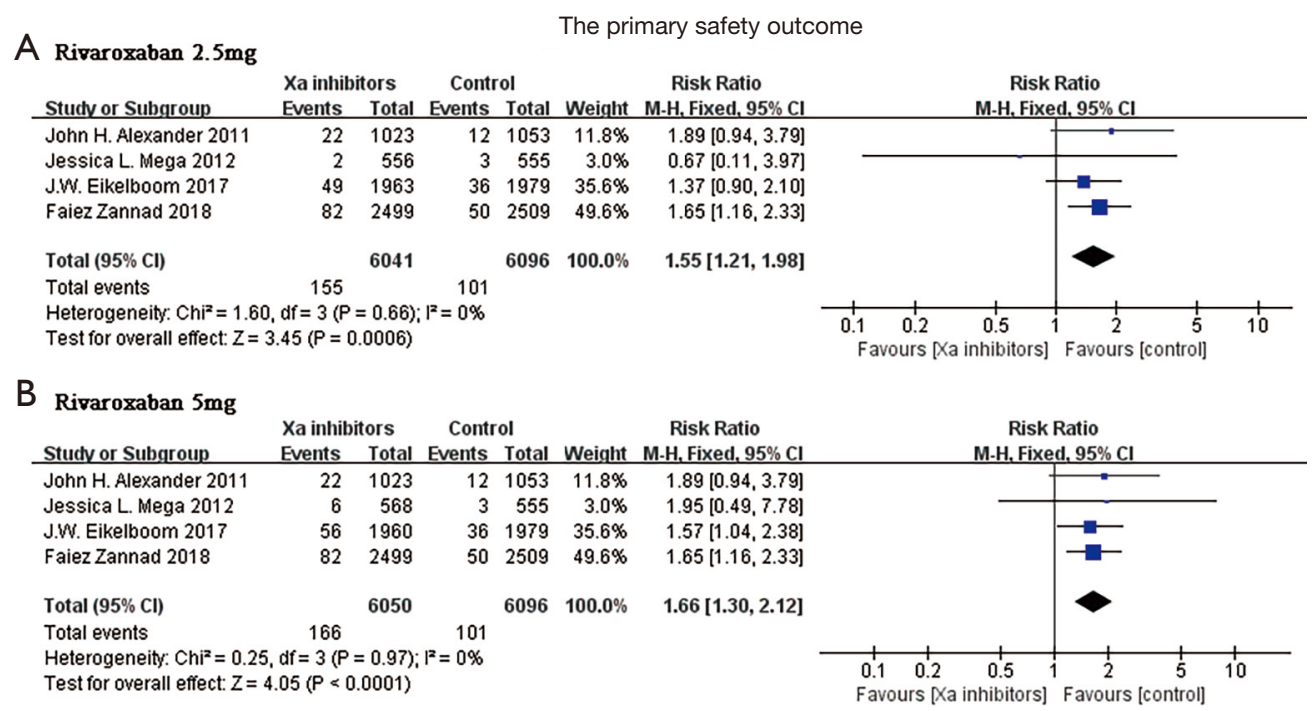

Figure 4 Meta-analysis for the primary safety outcome. (A) Includes the rivaroxaban $2.5 \mathrm{mg}$ group in the ATLAS ACS 2-TIMI 51 trial and rivaroxaban $2.5 \mathrm{mg}$ plus aspirin $100 \mathrm{mg}$ group in the COMPASS trial; (B) includes the rivaroxaban $5 \mathrm{mg}$ group in the ATLAS ACS 2-TIMI 51 trial and the rivaroxaban $5 \mathrm{mg}$ group in the COMPASS trial. ATLAS ACS 2-TIMI 51, Anti-Xa Therapy to Lower Cardiovascular Events in Addition to Standard Therapy in Subjects with Acute Coronary Syndrome-Thrombolysis in Myocardial Infarction 51. COMPASS, Cardiovascular Outcomes for People Using Anticoagulation Strategies.

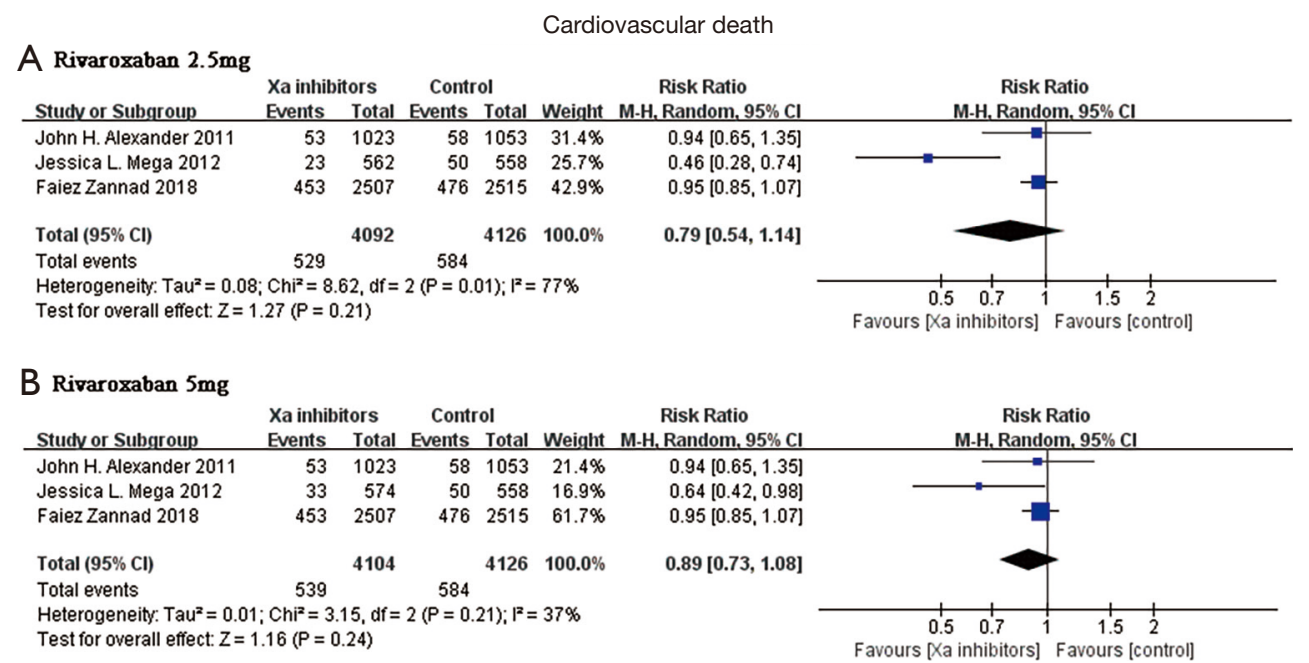

Figure 5 Meta-analysis for cardiovascular death. (A) includes the rivaroxaban $2.5 \mathrm{mg}$ group in the ATLAS ACS 2-TIMI 51 trial; (B) includes the rivaroxaban $5 \mathrm{mg}$ group in the ATLAS ACS 2-TIMI 51 trial. ATLAS ACS 2-TIMI 51, Anti-Xa Therapy to Lower Cardiovascular Events in Addition to Standard Therapy in Subjects with Acute Coronary Syndrome-Thrombolysis in Myocardial Infarction 51.

2-TIMI 51 trial, the results did not change (RR 0.93 , 95\% CI: 0.84-1.03, $\mathrm{P}=0.16)$. It did not make a difference to heterogeneity if the other three trials were excluded one at a time. Based on these results, we concluded that the ATLAS ACS 2-TIMI 51 trial was the source of heterogeneity. Regarding the sensitivity analysis involving the rivaroxaban $5 \mathrm{mg}$ groups, there was no significant change in heterogeneity. 

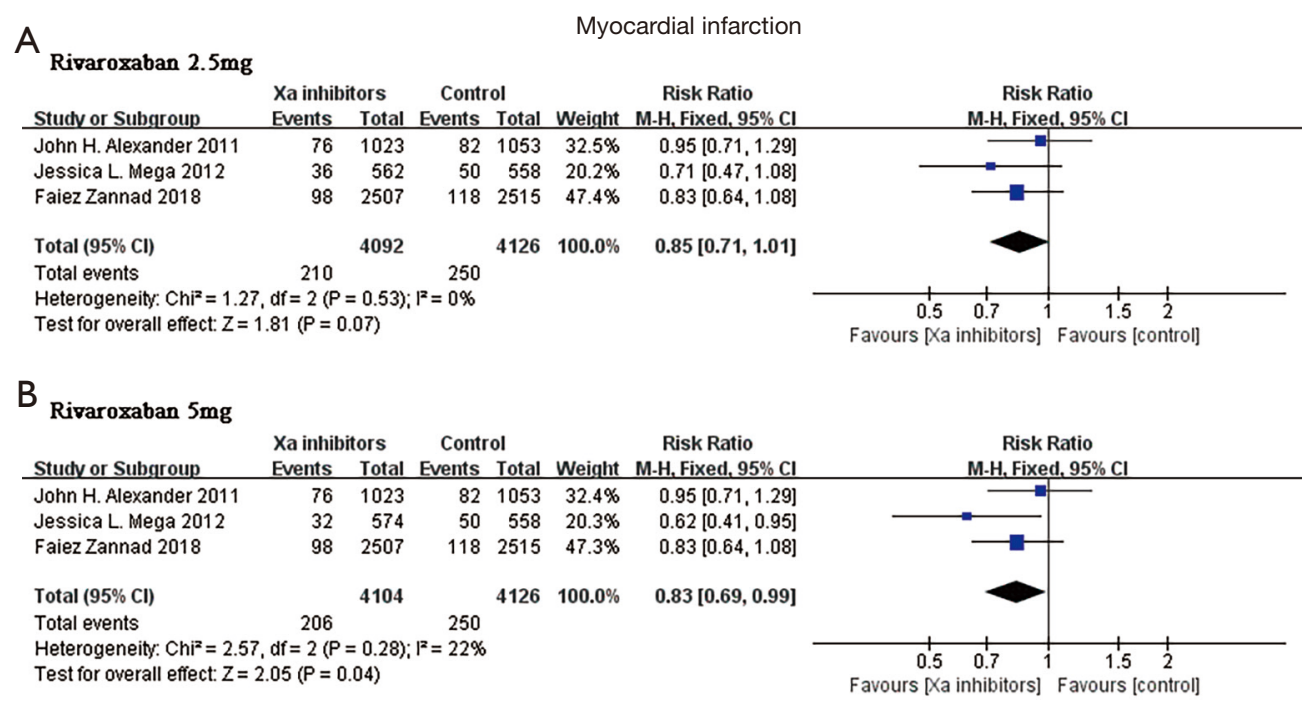

Figure 6 Meta-analysis for myocardial infarction. (A) includes the rivaroxaban $2.5 \mathrm{mg}$ group in the ATLAS ACS 2-TIMI 51 trial; (B) includes the rivaroxaban $5 \mathrm{mg}$ group in the ATLAS ACS 2-TIMI 51 trial. ATLAS ACS 2-TIMI 51, Anti-Xa Therapy to Lower Cardiovascular Events in Addition to Standard Therapy in Subjects with Acute Coronary Syndrome-Thrombolysis in Myocardial Infarction 51.

It was also found that there was apparent heterogeneity concerning comparisons of the $\mathrm{CV}$ death rates involving the rivaroxaban $2.5 \mathrm{mg}$ groups. When excluding the ATLAS ACS 2-TIMI 51 trial, heterogeneity decreased markedly $\left(\mathrm{I}^{2}=0 \%, \mathrm{P}\right.$ for heterogeneity 0.94$)$. However, there was still no significant difference between groups (RR 0.95, 95\% CI: $0.85-1.06, \mathrm{P}=0.40)$.

\section{Subgroup analysis}

Patients with ACS were eligible for the APPRAISE-2 and ATLAS ACS 2-TIMI 51 trials. However, the COMPASS trial only enrolled patients who had stable CV disease. The COMMANDER HF trial excluded patients who had acute MI. Therefore, we performed a subgroup analysis of patients with and without ACS. The results of the subgroup analysis were consistent with the combined group analysis for the primary efficacy outcome: for the rivaroxaban $2.5 \mathrm{mg}$ groups (non-ACS: RR 0.83, 95\% CI: $0.61-1.13, \mathrm{P}=0.24, \mathrm{I}^{2}=84 \%$, P for heterogeneity 0.01 ; ACS: RR $0.79,95 \%$ CI: $0.49-1.27$, $\mathrm{P}=0.33, \mathrm{I}^{2}=84 \%, \mathrm{P}$ for heterogeneity 0.01 , Figure $7 A$ ); and the rivaroxaban $5 \mathrm{mg}$ groups (Non-ACS: RR 0.90, 95\% CI: $0.76-1.06, \mathrm{P}=0.21, \mathrm{I}^{2}=52 \%$, P for heterogeneity 0.15; ACS: RR 0.81, $95 \%$ CI: $0.53-1.24, \mathrm{P}=0.33, \mathrm{I}^{2}=80 \%$, $\mathrm{P}$ for heterogeneity 0.02 , Figure $7 B$ ). While for the primary safety outcome: rivaroxaban $2.5 \mathrm{mg}$ groups did not increase significantly (RR 1.64, 95\% CI: $0.86-3.11, \mathrm{P}=0.13, \mathrm{I}^{2}=12 \%$,
P for heterogeneity 0.29 , Figure $7 C$ ) when compared with the control group. However, the results were different in that the safety in the ACS subgroup for the rivaroxaban $5 \mathrm{mg}$ groups (non-ACS: RR 1.61, 95\% CI: 1.24-2.11, $\mathrm{P}=0.0004, \mathrm{I}^{2}=0 \%, \mathrm{P}$ for heterogeneity 0.86 ; ACS: RR 1.90 , $95 \%$ CI: $1.02-3.54, \mathrm{P}=0.04, \mathrm{I}^{2}=0 \%, \mathrm{P}$ for heterogeneity 0.96, Figure 7D).

In the COMPASS trial, compared with aspirin alone, rivaroxaban with aspirin could significantly reduce the composite endpoints of cardiovascular death, $\mathrm{MI}$ and stroke (HR 0.67, 95\% CI: 0.52-0.87); and did not significantly increase bleeding event (HR 1.43, 95\% CI: 0.93-2.19).

\section{Discussion}

HF was associated with a prothrombotic or hypercoagulable state, which increases the risk of stroke and venous thromboembolism (23). Patients with dyskinesia in the left ventricle after MI were more likely to form mural thrombi and had an increased risk of subsequent peripheral thromboembolism and stroke (24). For patients with chronic atherosclerotic disease, coexisting HF could also lead to a higher incidence of major adverse cardiovascular events and mortality (25). Therefore, antithrombotic therapy was valuable among patients with $\mathrm{HF}$ and $\mathrm{CAD}$ or $\mathrm{PAD}$.

However, current guidelines in Europe and the United States recommend antithrombotic therapy only for HF 

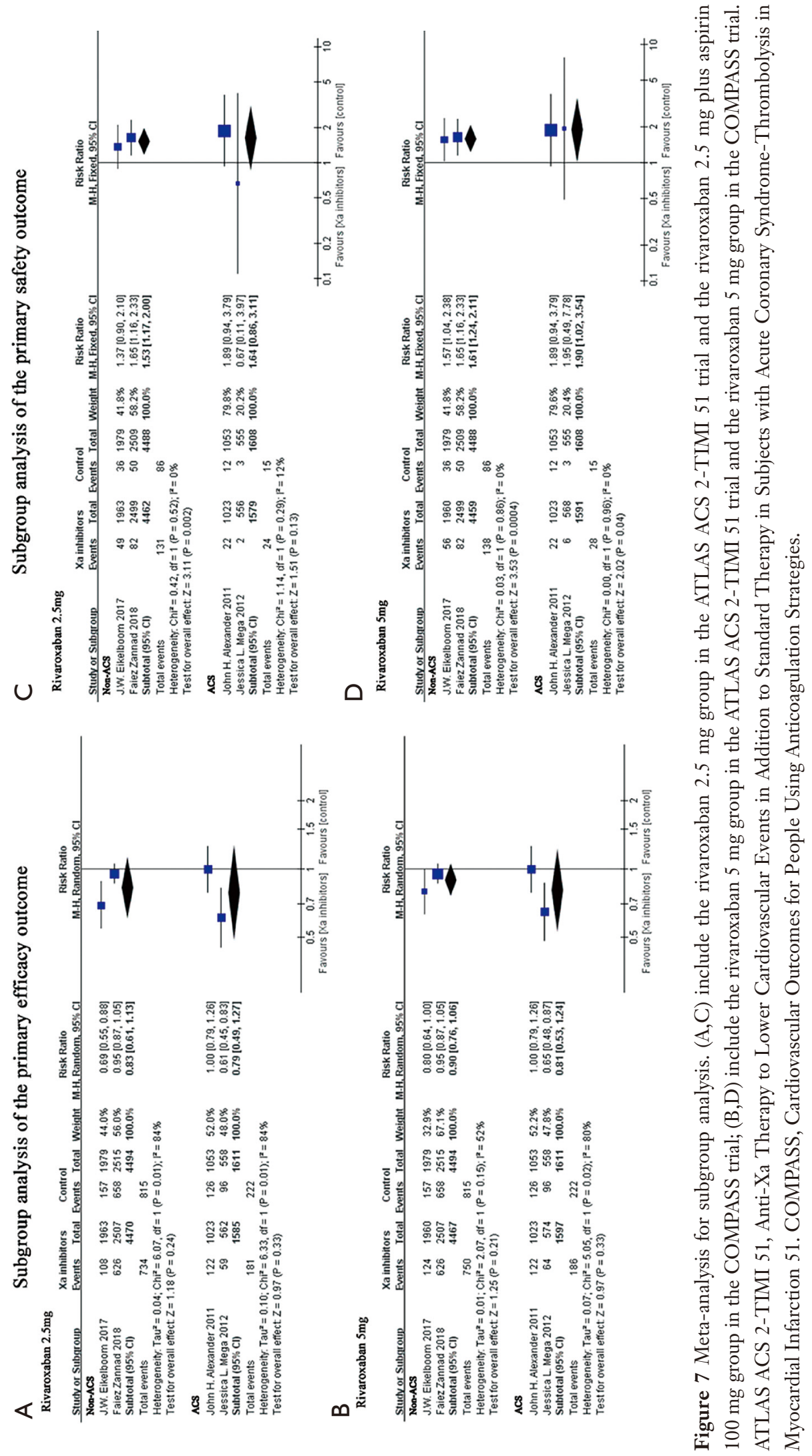
patients with a high risk of thromboembolism, such as left ventricular thrombosis or AF $(26,27)$. Previously, VKA was common in antithrombotic therapy. However, due to the slow onset of action, the narrow therapeutic concentration window, and interactions with multiple drugs and diet, VKA has many drawbacks in anticoagulation therapy (3). Furthermore, VKA does not perform well in patients with HF (28).

Rivaroxaban and apixaban are novel types of OACs and are direct-acting medications for activated factor $\mathrm{Xa}$, which is an integral component of the coagulation cascade. Factor Xa is the key protein that limits the propagation of thrombin generation. Thus Xa inhibitors play an important role in anticoagulation (29). A meta-analysis that included AF patients with HF showed that single- or high-dose Non-vitamin-K-dependent oral anti- coagulants (NOAC) regimens had a better efficacy and safety profile when compared with warfarin (30). Our study showed that Xa inhibitors reduced the primary efficacy outcome and CV death rate, while there were no statistically significant differences compared with the control groups.

A Rivaroxaban Once Daily Oral Direct Factor Xa Inhibitor Compared with Vitamin K Antagonism for Prevention of Stroke and Embolism Trial in Atrial Fibrillation (ROCKET AF) study performed by Halperin et al. (31) showed that compared with warfarin, rivaroxaban could reduce the incidence of stroke and other systemic circulatory embolism in patients with $\mathrm{AF}$, and bleeding events and other adverse events were comparable to warfarin. Furthermore, rivaroxaban could significantly reduce the incidence of intracranial hemorrhage and fatal hemorrhage. Not limited to AF, rivaroxaban is a better choice for the treatment of left ventricular thrombosis, with a better efficacy/safety ratio than warfarin (32). In addition, for the treatment of coronary or peripheral artery disease, low-dose rivaroxaban in combination with aspirin had a higher health cost effectiveness than aspirin alone (33).

Furthermore, our meta-analysis found that Xa inhibitors were associated with a higher risk of bleeding. This meta-analysis showed that the rivaroxaban $5 \mathrm{mg}$ group significantly decreased the incidence of MI; this outcome may be related to the fact that the $5 \mathrm{mg}$ rivaroxaban group had a lower risk of MI in the ATLAS ACS 2-TIMI 51 trial. In the subgroup analysis, there was no difference in the risk of bleeding for the rivaroxaban $2.5 \mathrm{mg}$ group and the apixaban group of ACS patients when compared to the control group. Therefore, rivaroxaban $2.5 \mathrm{mg}$ twice daily or apixaban $5 \mathrm{mg}$ twice daily should be recommended for patients with ACS and HF at high risk of bleeding, while rivaroxaban $5 \mathrm{mg}$ twice daily should be recommended for patients at high risk of MI. Besides, for patients with nonACS and HF at high risk of stroke, rivaroxaban $2.5 \mathrm{mg}$ twice daily (with or without aspirin $100 \mathrm{mg}$ once daily) could be an option.

According to the sensitivity analysis of the primary efficacy outcome and CV death rate, there was no statistical difference in the results before and after the analysis, which means that the conclusions are relatively stable.

In summary, the use of $\mathrm{Xa}$ inhibitors (alone or with aspirin) was associated with a higher risk of bleeding events when compared to placebo or aspirin alone, except for apixaban $5 \mathrm{mg}$ twice daily and rivaroxaban $2.5 \mathrm{mg}$ twice daily in patients with ACS. However, Xa inhibitors did not significantly reduce the risk of MACE. As for patients with ACS and HF at high risk of bleeding, rivaroxaban $2.5 \mathrm{mg}$ twice daily or apixaban $5 \mathrm{mg}$ twice daily may be an option. However, for patients at high risk of MI, rivaroxaban $5 \mathrm{mg}$ twice daily could be recommended. Besides, for patients without ACS and HF at high risk of stroke, the use of rivaroxaban $2.5 \mathrm{mg}$ twice daily (with or without aspirin $100 \mathrm{mg}$ once daily) could be an option. Therefore, Xa inhibitors should be used with caution in patients with $\mathrm{HF}$ and CAD or PAD according to the risk of MACE and bleeding.

There were several limitations to this study. Firstly, the definition of $\mathrm{HF}$ was inconsistent across the four trials included in this meta-analysis; trials involved patients with HF with varying degrees of severity. Secondly, rivaroxaban was chosen in all studies except for apixaban in the APPRAISE-2 trial regarding the types of Xa inhibitors. Thirdly, the clinical follow-up time was different among the four trials included. Fourthly, the kinds of antiplatelet drugs were also different in the trials. Fifthly, although the APPRAISE-2 trial only provided data on the event rate per 100 patient-years, the deviation was not large enough to affect the statistical difference shown in the result. Sixthly, although 14,694 participants were included in this metaanalysis, the number of included RCTs was small. Lastly, language restrictions might have resulted in the exclusion of eligible trials.

$\mathrm{Xa}$ inhibitors were associated with a higher risk of MACE and bleeding among HF and CAD or PAD patients. Therefore, $\mathrm{Xa}$ inhibitors should be used cautiously in patients with $\mathrm{HF}$ and $\mathrm{CAD}$ or PAD. 


\section{Acknowledgments}

Funding: None.

\section{Footnote}

Reporting Checklist: The authors have completed the PRISMA reporting checklist. Available at https://dx.doi. org/10.21037/apm-21-1645

Conflicts of Interest: All authors have completed the ICMJE uniform disclosure form (available at https://dx.doi. org/10.21037/apm-21-1645). The authors have no conflicts of interest to declare.

Ethical Statement: The authors are accountable for all aspects of the work in ensuring that questions related to the accuracy or integrity of any part of the work are appropriately investigated and resolved.

Open Access Statement: This is an Open Access article distributed in accordance with the Creative Commons Attribution-NonCommercial-NoDerivs 4.0 International License (CC BY-NC-ND 4.0), which permits the noncommercial replication and distribution of the article with the strict proviso that no changes or edits are made and the original work is properly cited (including links to both the formal publication through the relevant DOI and the license). See: https://creativecommons.org/licenses/by-nc-nd/4.0/.

\section{References}

1. Dunlay SM, Roger VL, Redfield MM. Epidemiology of heart failure with preserved ejection fraction. Nat Rev Cardiol 2017;14:591-602.

2. Gheorghiade M, Vaduganathan M, Fonarow GC, et al. Anticoagulation in heart failure: current status and future direction. Heart Fail Rev 2013;18:797-813.

3. Uretsky BF, Thygesen K, Armstrong PW, et al. Acute coronary findings at autopsy in heart failure patients with sudden death: results from the assessment of treatment with lisinopril and survival (ATLAS) trial. Circulation 2000;102:611-6.

4. Isnard R, Bauer F, Cohen-Solal A, et al. Non-vitamin $\mathrm{K}$ antagonist oral anticoagulants and heart failure. Arch Cardiovasc Dis 2016;109:641-50.

5. Cleland JG, Findlay I, Jafri S, et al. The Warfarin/Aspirin Study in Heart failure (WASH): a randomized trial comparing antithrombotic strategies for patients with heart failure. Am Heart J 2004;148:157-64.

6. Massie BM, Collins JF, Ammon SE, et al. Randomized trial of warfarin, aspirin, and clopidogrel in patients with chronic heart failure: the Warfarin and Antiplatelet Therapy in Chronic Heart Failure (WATCH) trial. Circulation 2009;119:1616-24.

7. Cokkinos DV, Haralabopoulos GC, Kostis JB, et al. Efficacy of antithrombotic therapy in chronic heart failure: the HELAS study. Eur J Heart Fail 2006;8:428-32.

8. Homma S, Thompson JL, Pullicino PM, et al. Warfarin and aspirin in patients with heart failure and sinus rhythm. N Engl J Med 2012;366:1859-69.

9. Khatib R, Ludwikowska M, Witt DM, et al. Vitamin K for reversal of excessive vitamin $\mathrm{K}$ antagonist anticoagulation: a systematic review and meta-analysis. Blood Adv 2019;3:789-96.

10. Liberati A, Altman DG, Tetzlaff J, et al. The PRISMA statement for reporting systematic reviews and metaanalyses of studies that evaluate healthcare interventions: explanation and elaboration. BMJ 2009;339:b2700.

11. Higgins JP, Altman DG, Gøtzsche PC, et al. The Cochrane Collaboration's tool for assessing risk of bias in randomised trials. BMJ. 2011 Oct 18;343:d5928.

12. Alexander JH, Lopes RD, James S, et al. Apixaban with antiplatelet therapy after acute coronary syndrome. $\mathrm{N}$ Engl J Med 2011;365:699-708.

13. Cornel JH, Lopes RD, James S, et al. Anticoagulant therapy and outcomes in patients with prior or acute heart failure and acute coronary syndromes: insights from the APixaban for PRevention of Acute ISchemic Events 2 trial. Am Heart J 2015;169:531-8.

14. Korjian S, Braunwald E, Daaboul Y, et al. Usefulness of Rivaroxaban for Secondary Prevention of Acute Coronary Syndrome in Patients With History of Congestive Heart Failure (from the ATLAS-ACS-2 TIMI-51 Trial). Am J Cardiol 2018;122:1896-901.

15. Zannad F, Anker SD, Byra WM, et al. Rivaroxaban in patients with heart failure, sinus rhythm, and coronary disease. N Engl J Med 2018;379:1332-42.

16. Branch KR, Probstfield JL, Eikelboom JW, et al. Rivaroxaban with or without aspirin in patients with heart failure and chronic coronary or peripheral artery disease. Circulation 2019;140:529-37.

17. Mega JL, Braunwald E, Wiviott SD, et al. Rivaroxaban in patients with a recent acute coronary syndrome. N Engl J Med 2012;366:9-19.

18. Gibson CM, Mega JL, Burton P, et al. Rationale and 
design of the Anti-Xa therapy to lower cardiovascular events in addition to standard therapy in subjects with acute coronary syndrome-thrombolysis in myocardial infarction 51 (ATLAS-ACS 2 TIMI 51) trial: a randomized, double-blind, placebo-controlled study to evaluate the efficacy and safety of rivaroxaban in subjects with acute coronary syndrome. Am Heart J 2011;161:815-21.e6.

19. Eikelboom JW, Connolly SJ, Bosch J, et al. Rivaroxaban with or without Aspirin in Stable Cardiovascular Disease. N Engl J Med 2017;377:1319-30.

20. Bosch J, Eikelboom JW, Connolly SJ, et al. Rationale, Design and Baseline Characteristics of Participants in the Cardiovascular Outcomes for People Using Anticoagulation Strategies (COMPASS) Trial. Can J Cardiol 2017;33:1027-35.

21. Zannad F, Greenberg B, Cleland JG, et al. Rationale and design of a randomized, double-blind, event-driven, multicentre study comparing the efficacy and safety of oral rivaroxaban with placebo for reducing the risk of death, myocardial infarction or stroke in subjects with heart failure and significant coronary artery disease following an exacerbation of heart failure: the COMMANDER HF trial. Eur J Heart Fail 2015;17:735-42.

22. Cumpston M, Li T, Page MJ, et al. Updated guidance for trusted systematic reviews: a new edition of the Cochrane Handbook for Systematic Reviews of Interventions. Cochrane Database Syst Rev 2019;10:ED000142.

23. Lip GY, Gibbs CR. Does heart failure confer a hypercoagulable state? Virchow's triad revisited. J Am Coll Cardiol 1999;33:1424-6.

24. Ferreira JP, Girerd N, Gregson J, et al. Stroke Risk in Patients With Reduced Ejection Fraction After Myocardial Infarction Without Atrial Fibrillation. J Am Coll Cardiol 2018;71:727-35.

25. Shah KS, Xu H, Matsouaka RA, et al. Heart failure with preserved, borderline, and reduced ejection fraction: 5-year outcomes. J Am Coll Cardiol 2017;70:2476-86.

26. Ponikowski P, Voors AA, Anker SD, et al. 2016 ESC Guidelines for the diagnosis and treatment of acute and

Cite this article as: Chen Y, Feng X, Qi C, Zhou L, Sun Y, Gu Z, Li X. Efficacy and safety of Xa inhibitors in patients with heart failure and coronary or peripheral artery disease: a systematic review and meta-analysis of randomized controlled trials. Ann Palliat Med 2021;10(7):8082-8093. doi: 10.21037/ apm-21-1645 chronic heart failure: The Task Force for the diagnosis and treatment of acute and chronic heart failure of the European Society of Cardiology (ESC)Developed with the special contribution of the Heart Failure Association (HFA) of the ESC. Eur Heart J 2016;37:2129-200.

27. Yancy CW, Jessup M, Bozkurt B, et al. 2017 ACC/ AHA/HFSA Focused Update of the 2013 ACCF/AHA Guideline for the Management of Heart Failure: A Report of the American College of Cardiology/American Heart Association Task Force on Clinical Practice Guidelines and the Heart Failure Society of America. Circulation 2017;136:e137-e161.

28. Cleland JG, Mumtaz S, Cecchini L. Role of antithrombotic agents in heart failure. Curr Cardiol Rep 2012;14:314-25.

29. Celikyurt I, Meier CR, Kuhne M, et al. Safety and Interactions of Direct Oral Anticoagulants with Antiarrhythmic Drugs. Drug Saf 2017;40:1091-8.

30. Xiong Q, Lau YC, Senoo K, et al. Non-vitamin K antagonist oral anticoagulants (NOACs) in patients with concomitant atrial fibrillation and heart failure: a systemic review and meta-analysis of randomized trials. Eur J Heart Fail 2015;17:1192-200.

31. Halperin JL, Hankey GJ, Wojdyla DM, et al. Efficacy and safety of rivaroxaban compared with warfarin among elderly patients with nonvalvular atrial fibrillation in the Rivaroxaban Once Daily, Oral, Direct Factor Xa Inhibition Compared With Vitamin K Antagonism for Prevention of Stroke and Embolism Trial in Atrial Fibrillation (ROCKET AF). Circulation 2014;130:138-46. Erratum in: Circulation 2018;138:e783.

32. Tomasoni D, Sciatti E, Bonelli A, et al. Direct Oral Anticoagulants for the Treatment of Left Ventricular Thrombus-A New Indication? A Meta-summary of Case Reports. J Cardiovasc Pharmacol 2020;75:530-4.

33. Cowie MR, Lamy A, Levy P, et al. Health economic evaluation of rivaroxaban in the treatment of patients with chronic coronary artery disease or peripheral artery disease. Cardiovasc Res 2020;116:1918-24.

(English Language Editors: B. Meiser and J. Chapnick) 


\section{Supplementary}

\section{Search terms}

(CAD ("coronary artery disease" or "coronary artery diseases" or "coronary disease" or "acute coronary syndrome" or "acute coronary syndromes" or "coronary arteriosclerosis" or "coronary arterioscleroses" or "myocardial ischemia" or "myocardial ischemias" or "ischemic heart disease" or "ischemic heart diseases" or "myocardial infarction" or "myocardial infarctions" or "cardiovascular stroke" or "cardiovascular strokes" or "heart attack" or "heart attackss" or "myocardial infarct" or "myocardial infarcts") OR PAD ("peripheral arterial disease" or "peripheral arterial diseases" or "peripheral angiopathy" or "peripheral angiopathies")) AND HF ("heart failure" or "heart decompensation" or "myocardial failure" or "congestive heart failure") AND Xa inhibitors ("factor Xa inhibitors" or "direct factor Xa inhibitors" or "Xa inhibitor" or "Xa inhibitors" or "rivaroxaban" or "xarelto" or "bay 597939 " or "edoxaban" or "edoxaban tosylate" or "DU-176" or "DU-176b" or "apixaban" or "BMS 562247 ")

\section{Registration number in PROSPERO}

CRD42019139777 